\title{
Lithiation and Side-Chain Substitution of 3-Alkyl-1H-quinoxalin-2-ones
}

\author{
Keith Smith, ${ }^{*}$ Gamal A. El-Hiti, ${ }^{1}$ Safaa A. Mahgoub \\ Centre for Clean Chemistry, Department of Chemistry, University of Wales Swansea, Singleton Park, Swansea SA2 8PP, UK \\ Fax: +44(1792)295261 \\ E-mail: k.smith@swansea.ac.uk \\ Received:
}

\begin{abstract}
Methyl-1H-quinoxalin-2-one has been doubly lithiated with $n$-butylithium at $-78{ }^{\circ} \mathrm{C}$ in THF. The dilithio reagent thus obtained reacts with various electrophiles (iodomethane, iodoethane, $\mathrm{D}_{2} \mathrm{O}$, benzaldehyde, benzophenone, cyclohexanone) to give modified 3 -substituted $1 H$-quinoxalin-2-ones in good yields. In the reaction of the dilithio reagent with phenyl isothiocyanate the product was a tautomer of the simple substitution product. Reaction of the dilithio reagent with iodine gives an oxidatively dimerised product instead of the 3-iodomethyl derivative. Lithiations of 3-ethyl- and 3-propyl-1 $H$-quinoxalin-2-ones, followed by reactions with representative electrophiles (benzaldehyde, benzophenone, cyclohexanone), behaved in a similar manner to give the corresponding modified 3 -substituted derivatives in good yields.
\end{abstract}

Key words: double lithiation, $1 H$-quinoxalin-2-ones, electrophiles, tautomerisation, oxidative dimerisation

Lithiation of aromatic compounds has provided an important synthetic alternative to electrophilic substitution reactions. $^{2}$ Various heterocycles have been lithiated by the use of lithium reagents at low temperatures. The organolithiums thus obtained in such reactions are very useful intermediates for the synthesis of more complex substituted heterocycles. ${ }^{3}$ The high reactivity of diazines towards nucleophilic reagents makes the lithiation of such derivatives more difficult than that of most heterocyclic compounds due to nucleophilic addition of the lithium reagent to the $\mathrm{C}=\mathrm{N}$ bond. However, successful lithiation of diazines has been achieved by the use of less nucleophilic lithium reagents such as LDA or LTMP. ${ }^{4}$ We have previously reported many examples of the synthetic application of organolithium compounds, ${ }^{5}$ and in particular on the syntheses of more complex 2substituted $3 H$-quinazolin-4-ones via directed lithiation. ${ }^{6}$
Lithiation of quinoxalines containing substituents that direct ortho-lithiation provides a method of producing substituted derivatives in moderate to good yields. ${ }^{7}$ In the course of the development of new synthetic methods for the synthesis of pharmaceutical intermediates, we became interested in $\alpha$-lithiation of 3 -alkyl-1Hquinoxalin-2-ones as a possible method for the synthesis of more complex modified substituted $1 H$-quinoxalin-2one derivatives. Compounds possessing the quinoxaline ring system have important agricultural and pharmaceutical applications. ${ }^{8}$ We now report on the successful synthesis of 3 -substituted $1 H$-quinoxalin-2-ones via lithiation of 3-alkyl-1H-quinoxalin-2-ones.

3-Methyl-1H-quinoxalin-2-one (1) was prepared according to the literature procedure. ${ }^{9}$ Double lithiation of $\mathbf{1}$ occurred smoothly and rapidly with $n$-butyllitium $(2.2$ equivalents) at $-78^{\circ} \mathrm{C}$ in THF. Initial addition of $n$-BuLi provided the monolithio reagent 2 as a yellow solution until approximately one equivalent had been added, then gave the dilithio reagent $\mathbf{3}$ as an orange solution as the remaining $n$-BuLi was added. Reactions of the dilithio reagent 3 with a variety of electrophiles (iodomethane, iodoethane, $\mathrm{D}_{2} \mathrm{O}$, benzaldehyde, benzophenone, cyclohexanone) gave the modified 3-substituted 
1H-quinoxalin-2-ones (4-9) (Scheme 1) in good yields (Table 1).

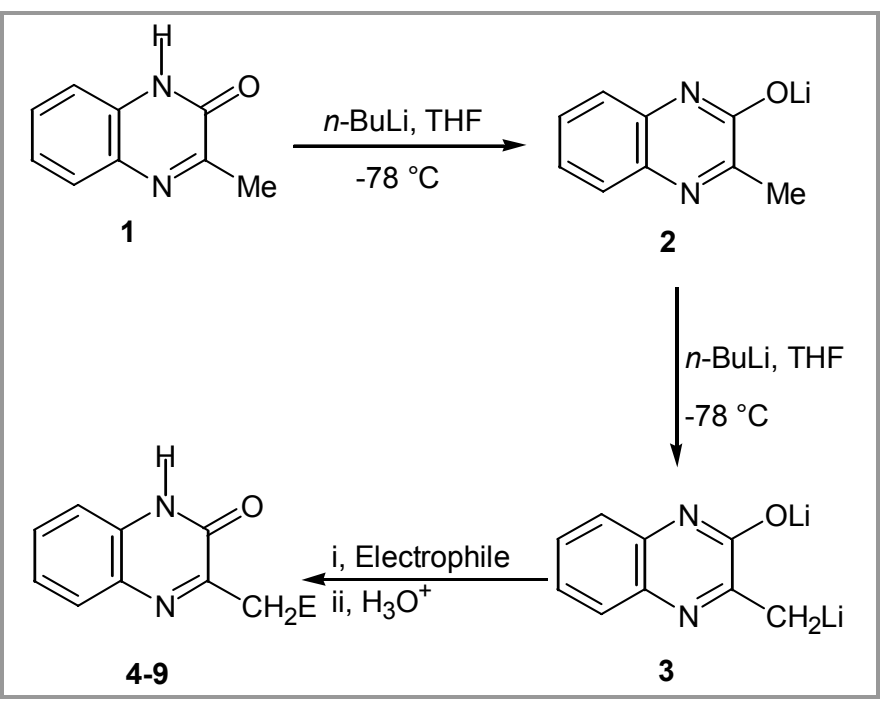

Scheme 1

\begin{tabular}{llll} 
Table 1 & \multicolumn{3}{l}{ Yields of products 4-9 according to Scheme 1} \\
\hline Product & Electrophile & $\mathrm{E}$ & Yield (\%) ${ }^{\mathrm{a}}$ \\
\hline $\mathbf{4}$ & $\mathrm{MeI}$ & $\mathrm{Me}$ & 77 \\
$\mathbf{5}$ & $\mathrm{EtI}$ & $\mathrm{Et}$ & 72 \\
$\mathbf{6}$ & $\mathrm{D}_{2} \mathrm{O}$ & $\mathrm{D}$ & 78 \\
$\mathbf{7}$ & $\mathrm{PhCHO}_{\mathbf{8}}$ & $\mathrm{PhCH}(\mathrm{OH})$ & 71 \\
$\mathbf{8}$ & $\mathrm{Ph}_{2} \mathrm{CO}$ & $\mathrm{Ph}_{2} \mathrm{C}(\mathrm{OH})$ & 80 \\
$\mathbf{9}$ &
\end{tabular}

As indicated in Table 1, the yields of isolated, purified products were good. No $N$-substitution was observed when an excess of iodomethane or iodoethane was used as electrophile.

Reaction of the dilithio reagent of compound $\mathbf{1}$ with phenyl isothiocyanate proceeded in an interesting manner to give compound $\mathbf{1 0}$ in $70 \%$ isolated yield (Scheme 2). Compound $\mathbf{1 0}$ is a tautomer of the simple substitution product (as would be obtained according to Scheme 1, where $\mathrm{E}$ is $\mathrm{PhNHCS})$.

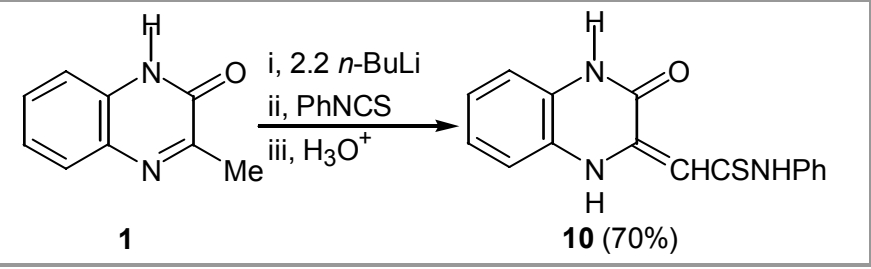

Scheme 2

The structure of compound $\mathbf{1 0}$ was confirmed by its NMR and mass spectra. The EI mass spectrum showed a molecular ion peak at $m / z 258$, and the CI mass spectrum showed a pseudo molecular ion peak at $m / z 259$, while the high resolution mass of the pseudo molecular ion confirmed the formula as $\mathrm{C}_{15} \mathrm{H}_{19} \mathrm{~N}_{2} \mathrm{O}_{2}\left(\mathrm{MH}^{+}\right)$. The ${ }^{1} \mathrm{H}$ NMR spectrum showed the presence of three exchangeable singlets due to three $\mathrm{NH}$ protons, resonating at $14.25,11.76$ and $11.21 \mathrm{ppm}$. The ${ }^{13} \mathrm{C}$ NMR spectrum showed six quaternary and eight $\mathrm{CH}$ carbons, whereas the simple substitution product would have shown six quaternary, seven $\mathrm{CH}$ and one $\mathrm{CH}_{2}$ carbons.

Reaction of the dilithio reagent of compound $\mathbf{1}$ with iodine as electrophile resulted in oxidative dimerisation to give compound $\mathbf{1 1}$ in $70 \%$ crude yield (Scheme 3). Compound 11 is highly insoluble in most solvents. After extraction of impurities with hot methanol, the yield was reduced to $63 \%$.

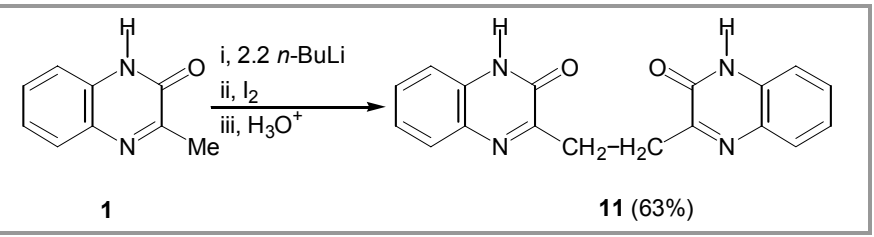

Scheme 3

The structure of compound $\mathbf{1 1}$ was confirmed by its ${ }^{1} \mathrm{H}$ NMR and mass spectra. The EI mass spectrum showed 
a molecular ion peak at $\mathrm{m} / \mathrm{z} 346$, while the high resolution mass of the molecular ion confirmed the formula as $\mathrm{C}_{18} \mathrm{H}_{14} \mathrm{~N}_{4} \mathrm{O}_{2}$. The ${ }^{1} \mathrm{H}$ NMR spectrum was consistent with the structure 11, but the material was too insoluble to give a good ${ }^{13} \mathrm{C}$ NMR spectrum.

Attention was next turned to the lithiation of 3-ethyl- $1 H$ quinoxalin-2-one (4) and 3-propyl-1 $H$-quinoxalin-2-one (5), which had been obtained by alkylation of the dilithio reagent 3 according to Scheme 1. If successful, this would suggest that the lithiation process was tolerant of a variety of primary alkyl groups at position 3 . It was found that successful lithiation of $\mathbf{4}$ and $\mathbf{5}$ was achieved using $n$-BuLi (2.2 equivalents) in THF at $-78{ }^{\circ} \mathrm{C}$ under nitrogen for $1 \mathrm{~h}$. The dilithio reagents thus obtained reacted with representative electrophiles (benzaldehyde, benzophenone, cyclohexanone) (Scheme 4) to give the corresponding modified derivatives $\mathbf{1 2 - 1 4}$ in good yields (Table 2).

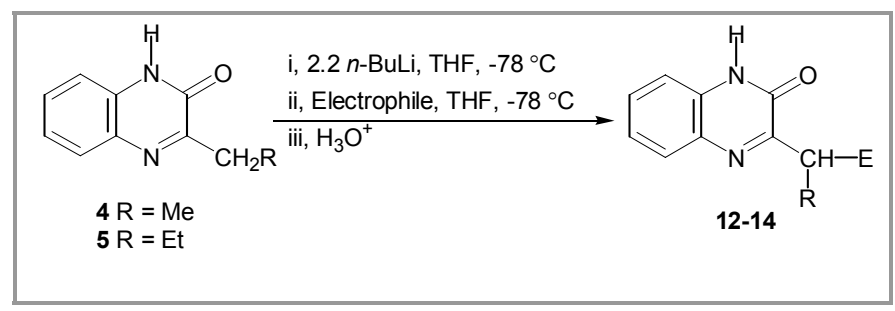

Scheme 4

\begin{tabular}{lllll} 
Table 2 & \multicolumn{3}{c}{ Yields of products 12-14 according to Scheme 4 } \\
\hline Product & $\mathrm{R}$ & Electrophile & $\mathrm{E}$ & Yield (\%) ${ }^{\mathrm{a}}$ \\
\hline $\mathbf{1 2}$ & $\mathrm{Me}$ & $\mathrm{PhCHO}$ & $\mathrm{PhCH}(\mathrm{OH})$ & 77 \\
$\mathbf{1 3}$ & $\mathrm{Me}$ & $\mathrm{Ph}_{2} \mathrm{CO}$ & $\mathrm{Ph}_{2} \mathrm{C}(\mathrm{OH})$ & 71 \\
$\mathbf{1 4}$ & $\mathrm{Et}$
\end{tabular}

The spectral characteristics of compounds 12-14 were consistent with the assigned structures. The NMR spectra of compound $\mathbf{1 2}$ showed the expected presence of two racemic diastereoisomers in unequal proportions.

In conclusion, this work describes a simple and convenient method for the high yield synthesis of more complex 3 -substituted $1 H$-quinoxalin-2-ones, which might have pharmacological activities and are difficult to prepare by other means.

Melting points were determined on an electrothermal digital melting point apparatus and are reported uncorrected. ${ }^{1} \mathrm{H}$ and ${ }^{13} \mathrm{C}$ NMR spectra were recorded on a Bruker spectrometer operating at $400 \mathrm{MHz}$ for ${ }^{1} \mathrm{H}$ and $100 \mathrm{MHz}$ for ${ }^{13} \mathrm{C}$ measurement. Chemical shifts are reported in parts per million relative to tetramethylsilane. Low-resolution mass spectra were recorded on a VG 12253 spectrometer, electron impact (E1) at $70 \mathrm{eV}$ and chemical ionization (CI) by use of ammonia as ionizing gas. Accurate mass data were obtained on a VG ZAB-E instrument. n-Butyllithium was obtained from Aldrich Chemical Company and was estimated prior to use by the method of Watson and Eastham. ${ }^{10}$ THF was distilled from sodium benzophenone ketyl. Other chemicals were obtained from Aldrich Chemical Company and used without further purification.

General procedure for the synthesis of modified 3substituted $1 \mathrm{H}$-quinoxalin-2-ones (4-14)

A solution of $n$-BuLi $(1.76 \mathrm{~mL}, 2.5 \mathrm{M}, 4.4 \mathrm{mmol})$ was added in a dropwise manner to a stirred solution of 3alkyl-1H-quinoxalin-2-one $(2.0 \mathrm{mmol})$ in dry THF $(60$ $\mathrm{mL}$ in the case of $\mathbf{1}$ and $20 \mathrm{~mL}$ in the cases of $\mathbf{4}$ and 5) maintained at $-78{ }^{\circ} \mathrm{C}$ under $\mathrm{N}_{2}$. Formation of the dilithio reagent was observed as an orange solution. The mixture was stirred at $-78^{\circ} \mathrm{C}$ under $\mathrm{N}_{2}$ for an additional $1 \mathrm{~h}$, after which an electrophile $(2.2 \mathrm{mmol})$, in dry THF (10 $\mathrm{mL}$ ) if solid, otherwise neat, was added. The mixture was stirred for $2 \mathrm{~h}$ at $-78{ }^{\circ} \mathrm{C}$ under $\mathrm{N}_{2}$, then removed from the cooling bath and allowed to warm to room temperature, diluted with ethyl acetate $(20 \mathrm{~mL})$ and quenched with aqueous ammonium chloride solution (20 $\mathrm{mL})$. The organic layer was washed with water $(2 \times 20$ $\mathrm{mL})$, dried $\left(\mathrm{MgSO}_{4}\right)$ and evaporated under reduced pressure. The products were recrystallised, subjected to column chromatography or washed with hot methanol to give the appropriate pure products 4-14.

\section{3-Ethyl-1H-quinoxalin-2-one (4)}

Recrystallised from aq. $\mathrm{MeOH}$; mp 190-191 ${ }^{\circ} \mathrm{C}$ (lit. ${ }^{11}$ $\left.191{ }^{\circ} \mathrm{C}\right)$. 


\section{3-Propyl-1H-quinoxalin-2-one (5)}

Recrystallised from aq. MeOH; mp $184-185{ }^{\circ} \mathrm{C}$ (lit. ${ }^{11}$ $\left.185^{\circ} \mathrm{C}\right)$.

\section{3-Deuteriomethyl-1H-quinoxalin-2-one (6)}

Recrystallised from MeOH; mp $245-247^{\circ} \mathrm{C}$.

${ }^{1} \mathrm{H}$ NMR (DMSO- $d_{6}$ ): $\delta=12.33$ (s, exch., $\left.1 \mathrm{H}\right), 7.66$ (d, $J=8.0 \mathrm{~Hz}, 1 \mathrm{H}), 7.45$ (apparent t, $J=8 \mathrm{~Hz}, 1 \mathrm{H}$ ), 7.277.22 (m, $2 \mathrm{H}), 2.37(1: 1: 1 \mathrm{t}, J=2 \mathrm{~Hz}, 2 \mathrm{H})$.

${ }^{13} \mathrm{C}$ NMR (DMSO- $d_{6}$ ): $\delta=159.5(\mathrm{~s}), 155.2(\mathrm{~s}), 132.2$ (s), 132.0 (s), 129.6 (d), 128.2 (d), 123.3 (d), 115.5 (d), 20.8, 20.6, $20.4(1: 1: 1 \mathrm{t})$.

EI-MS: $m / z(\%)=162\left(\mathrm{M}^{+}+1,9\right), 161\left(\mathrm{M}^{+}, 83\right), 131$ (96), 132 (100), 105 (15), 90 (42), 63 (38).

CI-MS: $m / z(\%)=179\left(\mathrm{M}^{+}+\mathrm{NH}_{4}, 6\right), 162\left(\mathrm{MH}^{+}, 100\right)$, 146 (47), 133 (9), 94 (8).

HRMS: $m / z$ calcd for $\mathrm{C}_{9} \mathrm{H}_{8} \mathrm{~N}_{2} \mathrm{OD}\left(\mathrm{MH}^{+}\right), 162.0777$; found, 162.0778 .

\section{3-(2-Hydroxy-2-phenylethyl)-1H-quinoxalin-2-one (7)}

Recrystallised from $\mathrm{MeOH} ; \mathrm{mp} 183-185^{\circ} \mathrm{C}$.

${ }^{1} \mathrm{H}$ NMR (DMSO- $\left.d_{6}\right): \delta=12.36$ (s, exch., $\left.1 \mathrm{H}\right), 7.75(\mathrm{dd}$, $J=8,1 \mathrm{~Hz}, 1 \mathrm{H}), 7.48$ (apparent dt $J=8,1 \mathrm{~Hz}, 1 \mathrm{H}$ ), 7.41 (d, $J=7 \mathrm{~Hz}, 2 \mathrm{H}), 7.32-7.20$ (m, $5 \mathrm{H}), 5.36$ (br s, exch., $1 \mathrm{H}), 5.28(\mathrm{t}, J=6.7 \mathrm{~Hz}, 1 \mathrm{H}), 3.12(\mathrm{~d}, J=6.7 \mathrm{~Hz}$, $2 \mathrm{H})$.

${ }^{13} \mathrm{C}$ NMR (DMSO- $\left.d_{6}\right): \delta=159.8(\mathrm{~s}), 155.3(\mathrm{~s}), 146.0(\mathrm{~s})$, 132.1 (s), 132.0 (s), 129.9 (d), 128.5 (d), 128.4 (d), 127.2 (d), 126.1 (d), 123.4 (d), 115.5 (d), 70.6 (d), 44.0 (t).

CI-MS: $m / z(\%)=267\left(\mathrm{MH}^{+}, 52\right), 249(21), 161$ (100), 147 (19), 138 (12), 119 (14), 105 (15), 94 (13).

HRMS: $m / z$ calcd for $\mathrm{C}_{16} \mathrm{H}_{15} \mathrm{~N}_{2} \mathrm{O}_{2}\left(\mathrm{MH}^{+}\right), 267.1133$; found, 267.1136 .

\section{3-(2-Hydroxy-2,2-diphenylethyl)-1H-quinoxalin-2- one (8)}

Recrystallised from $\mathrm{MeOH} ; \mathrm{mp} 200-201{ }^{\circ} \mathrm{C}$.

${ }^{1} \mathrm{H}$ NMR (DMSO- $d_{6}$ ): $\delta=12.56$ (s, exch., $\left.1 \mathrm{H}\right), 7.65$ (dd, $J=8,1 \mathrm{~Hz}, 1 \mathrm{H}), 7.55-7.44(\mathrm{~m}, 5 \mathrm{H}), 7.28-7.24$ (m, 6 $\mathrm{H}), 7.14$ (t, $J=7 \mathrm{~Hz}, 2 \mathrm{H}), 6.83$ (s, exch., $1 \mathrm{H}), 3.84$ (s, 2 $\mathrm{H})$.

${ }^{13} \mathrm{C}$ NMR (DMSO- $d_{6}$ ): $\delta=160.1(\mathrm{~s}), 155.6(\mathrm{~s}), 147.8(\mathrm{~s})$, 131.5 (s), 131.2 (s), 130.5 (d), 128.3 (d), 128.2 (d), 126.8 (d), 126.1 (d), 123.9 (d), 115.8 (d), $78.0(\mathrm{~s}), 42.7$ (t).

CI-MS: $m / z(\%)=343\left(\mathrm{MH}^{+}, 4\right), 325$ (11), 200 (34), 183 (100), 161 (14), 145 (23), 105 (34), 94 (44), 78 (25).

HRMS: $m / z$ calcd for $\mathrm{C}_{22} \mathrm{H}_{19} \mathrm{~N}_{2} \mathrm{O}_{2}\left(\mathrm{MH}^{+}\right), 343.1446$; found 343.1449 .

\section{3-[(1-Hydroxycyclohexyl)methyl]-1H-quinoxalin-2- one (9)}

Recrystallised from $\mathrm{MeOH} ; \mathrm{mp} 174-175^{\circ} \mathrm{C}$.

${ }^{1} \mathrm{H}$ NMR (DMSO- $d_{6}$ ): $\delta=12.42$ (s, exch., $1 \mathrm{H}$ ), 7.73 (dd, $J=8,1 \mathrm{~Hz}, 1 \mathrm{H}$ ), 7.48 (apparent dt, $J=8,1 \mathrm{~Hz}, 1 \mathrm{H}$ ), 7.30-7.25 (m, $2 \mathrm{H}), 4.66$ (s, exch., $1 \mathrm{H}), 2.97$ (s, $2 \mathrm{H})$, 1.60-1.51 (m, $4 \mathrm{H}), 1.43-1.12(\mathrm{~m}, 6 \mathrm{H})$.

${ }^{13} \mathrm{C}$ NMR (DMSO- $\left.d_{6}\right): \delta=160.2(\mathrm{~s}), 156.1(\mathrm{~s}), 132.0(\mathrm{~s})$, 131.9 (s), 130.0 (d), 128.5 (d), 123.5 (d), 115.6 (d), 71.5 $(\mathrm{s}), 45.1(\mathrm{t}), 37.8(\mathrm{t}), 25.7(\mathrm{t}), 20.0(\mathrm{t})$.

EI-MS: $m / z(\%)=258\left(\mathrm{M}^{+}, 5\right), 240(10), 160$ (93), 132 (90), 131 (100), 121 (41), 107 (54), 93 (73), 91 (89), 81 (70), 79 (78), 67 (91).

CI-MS: $m / z(\%)=259\left(\mathrm{MH}^{+}, 33\right), 241(13), 178(7), 161$ (100), 147 (12), 132 (8), 116 (49), 98 (12).

HRMS: $m / z$ calcd for $\mathrm{C}_{15} \mathrm{H}_{19} \mathrm{~N}_{2} \mathrm{O}_{2}\left(\mathrm{MH}^{+}\right), 259.1446$; found, 259.1446.

\section{3-(2-Phenylamino-2-thioxoethylidene)-3,4-dihydro- 1H-quinoxalin-2-one (10)}

Recrystallised from $\mathrm{MeOH} ; \mathrm{mp} 240-242^{\circ} \mathrm{C}$.

${ }^{1} \mathrm{H}$ NMR (DMSO- $d_{6}$ ): $\delta=14.25$ (s, exch., $\left.1 \mathrm{H}\right), 11.76(\mathrm{~s}$, exch., $1 \mathrm{H}), 11.21$ (s, exch., $1 \mathrm{H}), 7.83-7.62$ (m, $2 \mathrm{H})$, 7.39 (apparent t, $J=7.6 \mathrm{~Hz}, 2 \mathrm{H}), 7.28-7.18(\mathrm{~m}, 2 \mathrm{H})$, 7.12-7.04 (m, $3 \mathrm{H}), 6.58(\mathrm{~s}, 1 \mathrm{H})$.

${ }^{13} \mathrm{C}$ NMR (DMSO- $\left.d_{6}\right): \delta=188.2(\mathrm{~s}), 157.1(\mathrm{~s}), 142.0(\mathrm{~s})$, 139.7 (s), 128.9 (d), 126.2 (s), 125.8 (d), 125.7 (d), 124.4 (s), 124.0 (d), 123.8 (d), 123.2 (d), 115.6 (d), 115.5 (d).

EI-MS: $m / z(\%)=295\left(\mathrm{M}^{+}, 19\right), 262(41), 202$ (22), 174 (10), 160 (40), 131 (48), 93 (81), 77 (100), 65 (66), 51 (76).

CI-MS: $m / z(\%)=296\left(\mathrm{MH}^{+}, 20\right), 262(18), 166(24)$, 152 (100), 147 (47), 120 (72), 94 (56).

HRMS: $m / z$ calcd for $\mathrm{C}_{16} \mathrm{H}_{14} \mathrm{SN}_{3} \mathrm{O}\left(\mathrm{MH}^{+}\right), 296.0857$; found, 296.0855 .

\section{3,3'-(1,2-Ethanediyl)bis-1H-quinoxalin-2-one (11)}

The product was only sparingly soluble in organic solvents. It was washed thoroughly with hot methanol; mp 305-307 ${ }^{\circ} \mathrm{C}$.

${ }^{1} \mathrm{H}$ NMR (DMSO- $d_{6}$ ): $\delta=12.11$ (s, exch., $2 \mathrm{H}$ ), 7.45 (dd, $J=8,1 \mathrm{~Hz}, 2 \mathrm{H}), 7.24$ (apparent dt, $J=8,1 \mathrm{~Hz}, 2 \mathrm{H}$ ), 7.07-7.01 (m, $4 \mathrm{H}), 3.04$ (s, $4 \mathrm{H})$.

EI-MS: $m / z(\%)=319\left(\mathrm{M}^{+}+1,5\right), 318\left(\mathrm{M}^{+}, 16\right), 279$ (2), 185 (3), 173 (7), 160 (12), 145 (17), 131 (49), 104 (38), 90 (76), 77 (100), 63 (79).

HRMS: $m / z$ calcd for $\mathrm{C}_{18} \mathrm{H}_{14} \mathrm{~N}_{4} \mathrm{O}_{2}\left(\mathrm{M}^{+}\right), 318.1117$; found, 318.1113 . 


\section{3-(2-Hydroxy-1-methyl-2-phenylethyl)-1 $H$ - quinoxalin-2-one (12)}

Purified by column chromatography (silica gel; $\mathrm{Et}_{2} \mathrm{O}-$ hexane, 2:1); $\mathrm{mp} 156-158^{\circ} \mathrm{C}$.

Compound 12 appears in its NMR spectra as a mixture of two isomers, $\mathbf{a}$ and $\mathbf{b}$, in a ratio of 5:2. ${ }^{1} \mathrm{H}$ NMR (DMSO- $d_{6}$ ): 12a, $\delta=12.29$ (s, exch., $\left.1 \mathrm{H}\right), 7.75(\mathrm{~d}, J=8$ $\mathrm{Hz}, 1 \mathrm{H}), 7.47$ (apparent t, $J=8 \mathrm{~Hz}, 1 \mathrm{H}), 7.40-7.22(\mathrm{~m}$, $6 \mathrm{H}), 7.15(\mathrm{t}, J=7 \mathrm{~Hz}, 1 \mathrm{H}), 5.27(\mathrm{~d}, J=5 \mathrm{~Hz}$, exch., 1 $\mathrm{H}), 5.10$ (apparent t, $J=5 \mathrm{~Hz}, 1 \mathrm{H}), 3.70(\mathrm{~m}, 1 \mathrm{H}), 1.19$ $(\mathrm{d}, J=7 \mathrm{~Hz}, 3 \mathrm{H}) ; \mathbf{1 2 b}, \delta=12.29$ (s, exch., $1 \mathrm{H}$ ), 7.75 $(\mathrm{d}, J=8 \mathrm{~Hz}, 1 \mathrm{H}), 7.47$ (apparent t $J=8 \mathrm{~Hz}, 1 \mathrm{H}), 7.40$ $7.22(\mathrm{~m}, 6 \mathrm{H}), 7.15(\mathrm{t}, J=7 \mathrm{~Hz}, 1 \mathrm{H}), 5.23(\mathrm{~d}, J=4 \mathrm{~Hz}$, exch., $1 \mathrm{H}), 4.98(\mathrm{dd}, J=4 \& 9 \mathrm{~Hz}, 1 \mathrm{H}), 3.70(\mathrm{~m}, 1 \mathrm{H})$, $0.90(\mathrm{~d}, J=7 \mathrm{~Hz}, 3 \mathrm{H})$.

${ }^{13} \mathrm{C}$ NMR (DMSO- $\left.d_{6}\right):$ 12a, $\delta=163.6(\mathrm{~s}), 154.6(\mathrm{~s})$, $145.1(\mathrm{~s}), 131.9(\mathrm{~s}), 131.8(\mathrm{~s}), 129.8(\mathrm{~d}), 128.6(\mathrm{~d}), 128.0$ (d), 127.3 (d), 126.5 (d), 123.4 (d), 115.45 (d), 73.7 (d), 43.5 (d), 13.2 (q); 12b, $\delta=164.6$ (s), 155.1 (s), 144.7 (s), $132.1(\mathrm{~s}), 131.8(\mathrm{~s}), 129.8(\mathrm{~d}), 128.5(\mathrm{~d}), 128.3(\mathrm{~d}), 127.5$ (d), 126.9 (d), 123.4 (d), 115.49 (d), 76.4 (d), 43.4 (d), $15.7(\mathrm{q})$.

EI-MS: $m / z(\%)=281\left(\mathrm{M}^{+}+1,3\right), 280\left(\mathrm{M}^{+}, 1\right), 238(9)$, 174 (100), 145 (96), 131 (19), 105 (60), 77 (78).

CI-MS: $m / z(\%)=281\left(\mathrm{MH}^{+}, 20 \%\right), 263(6), 192(5)$, 175 (100), 147 (9), 105 (4).

HRMS: $m / z$ calcd for $\mathrm{C}_{17} \mathrm{H}_{17} \mathrm{~N}_{2} \mathrm{O}_{2}\left(\mathrm{MH}^{+}\right), 281.1290$; found, 281.1288 .

\section{3-(2-Hydroxy-1-methyl-2,2-diphenylethyl)-1H- quinoxalin-2-one (13)}

Recrystallised from EtOAc-Et $2 \mathrm{O}(1: 1) ; \mathrm{mp} 177-178^{\circ} \mathrm{C}$.

${ }^{1} \mathrm{H}$ NMR (DMSO- $d_{6}$ ): $\delta=12.72$ (s, exch., $1 \mathrm{H}$ ), 7.75$7.65(\mathrm{~m}, 5 \mathrm{H}), 7.53$ (apparent dt, $J=8,1 \mathrm{~Hz}, 1 \mathrm{H}), 7.45$ $7.32(\mathrm{~m}, 4 \mathrm{H}), 7.24$ (apparent t, $J=8 \mathrm{~Hz}, 1 \mathrm{H}), 7.17(\mathrm{t}, J$ $=8.0 \mathrm{~Hz}, 2 \mathrm{H}), 7.03$ (apparent t, $J=8,1 \mathrm{H}), 6.07(\mathrm{~s}$, exch., $1 \mathrm{H}), 4.96$ (q, $J=7 \mathrm{~Hz}, 1 \mathrm{H}), 1.32(\mathrm{~d}, J=7 \mathrm{~Hz}, 3$ $\mathrm{H})$.

${ }^{13} \mathrm{C}$ NMR (DMSO- $\left.d_{6}\right): \delta=165.8(\mathrm{~s}), 156.6(\mathrm{~s}), 148.9(\mathrm{~s})$, 146.4 (s), 132.1 (s), 130.9 (d), 128.6 (d), 128.5 (d), 126.8 (d), 126.1 (d), 125.0 (d), 116.3 (d), 80.8 (s), 41.0 (d), 15.4 (q).

EI-MS: $m / z(\%)=357\left(\mathrm{M}^{+}+1,12\right), 356\left(\mathrm{M}^{+}, 32\right), 339$ (10), 238 (19), 223 (100).

CI-MS: m/z (\%): $357\left(\mathrm{MH}^{+}, 80\right), 339$ (100).

HRMS: $m / z$ calcd for $\mathrm{C}_{23} \mathrm{H}_{21} \mathrm{~N}_{2} \mathrm{O}_{2}\left(\mathrm{MH}^{+}\right), 357.1603$; found, 357.1608 .

\section{3-[1-(1-Hydroxycyclohexyl)propyl]-1H-quinoxalin-2- one (14)}

Recrystallised from EtOAc-Et $2 \mathrm{O}(1: 1) ; \mathrm{mp} 156-157^{\circ} \mathrm{C}$.
${ }^{1} \mathrm{H}$ NMR (DMSO- $\left.d_{6}\right) \delta 12.41$ (s, exch., $\left.1 \mathrm{H}\right), 7.76$ (dd, $J$ $=8,1 \mathrm{~Hz}, 1 \mathrm{H}), 7.49$ (apparent dt, $J=8,1 \mathrm{~Hz}, 1 \mathrm{H}$ ), 7.31-7.24 (m, $2 \mathrm{H}), 4.43$ (s, exch., $1 \mathrm{H}), 3.47$ (br, $1 \mathrm{H})$, $1.90-0.95(\mathrm{~m}, 12 \mathrm{H}), 0.67(\mathrm{t}, J=7 \mathrm{~Hz}, 3 \mathrm{H})$.

${ }^{13} \mathrm{C}$ NMR (DMSO- $\left.d_{6}\right): \delta=164.1(\mathrm{~s}), 156.1(\mathrm{~s}), 131.9(\mathrm{~s})$, $131.6(\mathrm{~s}), 130.1$ (d), 128.7 (d), 123.6 (d), 115.6 (d), 73.0 $(\mathrm{s}), 41.7(\mathrm{~d}), 35.5(\mathrm{t}), 25.3(\mathrm{t}), 21.7(\mathrm{t}), 21.6(\mathrm{t}), 13.1(\mathrm{q})$.

EI-MS: $m / z(\%)=386\left(\mathrm{M}^{+}, 11\right), 188(100), 173(44), 160$ (16), 81 (19), 65 (33).

CI-MS: $m / z(\%)=287\left(\mathrm{MH}^{+}, 91\right), 269$ (35), $247(43)$, 189 (100), $116(24)$.

HRMS: $m / z$ calcd for $\mathrm{C}_{17} \mathrm{H}_{23} \mathrm{~N}_{2} \mathrm{O}_{2}\left(\mathrm{MH}^{+}\right), 287.1759$; found, 287.1755 .

\section{Acknowledgments}

We thank the EPSRC Mass Spectroscopy Service, University of Wales Swansea, for recording the mass spectra. We also thank the EPSRC, the Higher Education Funding Council for Wales (ELWaHEFCW) and the University of Wales Swansea for grants that enabled the purchase and upgrading of NMR equipment used in the course of this work. G. A. El-Hiti thanks the University of Wales Swansea for financial support and the Royal Society of Chemistry for an international author grant.

\section{References}

(1) Permanent address: G. A. El-Hiti, Department of Chemistry, Faculty of Science, Tanta University, Tanta 31527, Egypt.

(2) (a) Slocum, D. W.; Jennings, C. A. J. Org. Chem. 1976, 41, 3653. (b) Gschwend, H. W.; Rodriguez, H. R. Org. React. 1979, 26, 1. (c) Beak, P.; Zajdel, W. J.; Reitz, D. B. Chem. Rev. 1984, 84, 471. (d) Führer, W.; Gschwend, H. W. J. Org. Chem. 1979, 44, 1133. (e) Snieckus, V. Chem. Rev. 1990, 90, 879. (f) Anctil, E. J.-G.; Snieckus, V. J. Organomet. Chem., 2002, 653, 150.

(3) (a) Marsais, F.; Quéguiner, G.; Snieckus, V.; Epsztajn, J. Adv. Heterocycl. Chem. 1991, 52, 187. (b) Turck, A.; Plé, N.; Quéguiner, G. Heterocycles 1994, 37, 2149. (c) El-Hiti, G. A. Heterocycles 2000, 53, 1839.

(4) (a) Gros, Ph.; Choppin, S.; Fort, Y. J. Org. Chem. 2003, 68, 2243. (b) Katritzky, A. R.; Manju, K.; Steel, P. J. J. Org. Chem. 2003, 68, 407. (c) Toudic, F.; Plé, N.; Turck, A.; Quéguiner, G. Tetrahedron 2002, 58, 283. (d) Rhonnstad, P.; Wensbo, D. Tetrahedron Lett. 2002, 43, 3137. (e) Mukherjee, C.; De, A. Synlett 2002, 325. (f) Gros, Ph.; Louërat, F.; Fort, Y. Org. Lett. 2002, 4, 1759. (g) Cuperly, D.; Gros, Ph.; Fort, Y. J. Org. Chem. 2002, 67, 238. (h) Gros, Ph.; Choppin, S.; Fort, Y. J. Org. Chem. 2002, 67, 2243. (i) Gros, Ph.; Choppin, S.; Mathieu, J.; Fort, Y. Eur. J. Org. Chem. 2002, 20, 3325. (j) Kondo, Y.; Asai, M.; Miura, T.; Uchiyama, M.; Sakamoto, T. Org. Lett. 2001, 3, 13. (k) Choppin, S.; Gros, Ph.; Fort, Y. Org. Lett. 2000, 2, 803. (1) Pollet, P.; Turck, A.; Plé, N.; Quéguiner, G. J. Org. Chem. 1999, 64, 4512.

(5) See for example: (a) Smith, K.; El-Hiti, G. A.; Hamilton, A. J. Chem. Soc., Perkin Trans. 1 1998, 4041. (b) Smith, K.; El-Hiti, G. A.; Pritchard, G. J.; Hamilton, A. J. Chem. Soc., Perkin Trans. 1 1999, 2299. (c) Smith, K.; El-Hiti, G. A.; Shukla, A. P. J. Chem. Soc., Perkin Trans. 1 1999, 2305. (d) Smith, K.; El-Hiti, G. A.; Hawes, A. C. Synlett 1999, 945 .

(6) a) Smith, K.; El-Hiti, G. A.; Abdo, M. A.; Abdel-Megeed, M. F. J. Chem. Soc., Perkin Trans. 1 1995, 1029. (b) Smith, 
K.; El-Hiti, G. A.; Abdel-Megeed, M. F.; Abdo, M. A. J. Org. Chem. 1996, 61, 647. (c) Smith, K.; El-Hiti, G. A.; Abdel-Megeed, M. F.; Abdo, M. A. J. Org. Chem. 1996, 61, 656. (d) Smith, K.; El-Hiti, G. A.; Abdel-Megeed, M. F.; Abdo, M. A. Collect. Czech. Chem. Commun. 1999, 64, 515. (e) Smith, K.; El-Hiti, G. A.; Abdel-Megeed, M. F. Russ. J. Org. Chem. 2003, accepted.

(7) (a) Kaiser, E. M. Tetrahedron 1983, 39, 2055. (b) Turck, A.; Plé, N.; Tallon, V.; Quéguiner, G. J. Heterocycl. Chem. 1993, 30, 1491. (c) Gautheron-Chapoulaud, V.; Salliot, I.; Plé, N.; Turck, A.; Quéguiner, G. Tetrahedron 1999, 55, 5389. (d) Parra, S.; Laurent, F.; Subra, G.; DeleuzeMasquefa, C.; Benezch, V.; Fabreguettes, J.-R.; Vidal, J.-P.; Pocock, T.; Elliott, K.; Small, R.; Escale, R.; Michel, A.; Chapat, J.-P.; Bonnet, P.-A. Eur. J. Med. Chem. 2001, 36, 255.

(8) See for example: (a) Asano, K.; Asai, S. Yakugaku Zasshi 1959, 79, 567; Chem. Abstr. 1959, 53, 21979. b) Hofmann, C. W.; Krajeurski, J. J.; Kutz, Ph. J.; Traxler, J. T.; Ristich, S. S. J. Agric. Food Chem. 1971, 1, 298. (c) Bucchini, D.; Fiszman, M.; Girad, M. Intervirology 1974, 3, 281. (d) Spada, A. P.; He, W.; Myers, M. R. PCT Int. Appl. WO 3149, 2000; Chem. Abstr. 2000, 133, 17477. (e) Myers, M. R.; Spada, A. P.; Persons, P. E.; Maguire, M. P. PCT Int. Appl. WO 3150, 2000; Chem. Abstr. 2000, 133, 17478. (f) Myers, M. R.; He, W.; Spada, A. P. PCT Int. Appl. WO 3151, 2000; Chem. Abstr. 2000, 133, 17479.

(9) Morrison, D. C. J. Am. Chem. Soc. 1954, 76, 4483.

(10) Watson, S. C.; Eastham J. F. J. Organomet. Chem. 1967, 9, 165.

(11) Tennant, G. J. Chem. Soc. C 1966, 2285. 
Lithiation and Side-Chain Substitution of 3-Alkyl-1H-quinoxalin-2-ones

K. Smith,* G. A. El-Hiti, S. A. Mahgoub

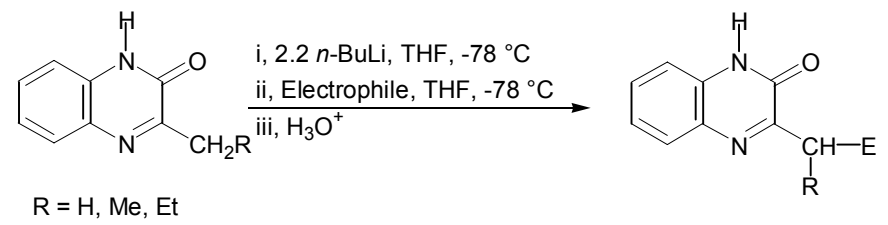

$\mathrm{R}=\mathrm{H}, \mathrm{Me}, \mathrm{Et}$

Lithiation of 3-Alkyl-1H-quinoxalin-2-ones 